\title{
Computed Tomography Using a Low Tube Voltage Technique for Acute Ischemic Stroke
}

\section{Kenji Nakamura1 ${ }^{*}$, Katsuhiko Maeda1, Masao Tanooka², Shuhei Aoyama1, Reiichi Ishikura ${ }^{3}$, Noriko Kotoura ${ }^{1}$}

\author{
${ }^{1}$ Department of Radiological Technology, Hyogo College of Medicine College Hospital, Nishinomiya, Japan \\ ${ }^{2}$ Department of Radiotherapy, Takarazuka City Hospital, Takarazuka, Japan \\ ${ }^{3}$ Department of Radiology, Hyogo College of Medicine, Nishinomiya, Japan \\ Email: ^hotatom8@yahoo.co.jp
}

How to cite this paper: Nakamura, K. Maeda, K., Tanooka, M., Aoyama, S., Ishikura, R. and Kotoura, N. (2019) Computed Tomography Using a Low Tube Voltage Technique for Acute Ischemic Stroke. $A d$ vances in Computed Tomography, 8, 24-35. https://doi.org/10.4236/act.2019.82003

Received: March 9, 2019

Accepted: June 17, 2019

Published: June 20, 2019

Copyright $\odot 2019$ by author(s) and Scientific Research Publishing Inc. This work is licensed under the Creative Commons Attribution International License (CC BY 4.0).

http://creativecommons.org/licenses/by/4.0/

\begin{abstract}
Computed tomography (CT) is commonly used to assess for cerebral hemorrhage and acute ischemic stroke. We investigated the accuracy of CT using a low tube voltage technique in acute ischemic stroke. We compared the standard deviation (SD), contrast between gray and white matter, and contrast-to-noise ratio (CNR) between three groups $(120 \mathrm{kV} 500 \mathrm{mAs}, 100 \mathrm{kV}$ $850 \mathrm{mAs}$, and $100 \mathrm{kV} 750 \mathrm{mAs}$ using hybrid iterative reconstruction) in 50 patients without lesions, and visual evaluation using the normalized rank approach was also performed. The mean value of SD was 4.02, 4.22, and 4.04, respectively, and the contrast between gray and white matter was $7.08,8.66$, and $8.68 \mathrm{HU}$, respectively; in addition, the CNR was $1.77,2.06$, and 2.15 , respectively. The difference between the $100 \mathrm{kV}$ and $120 \mathrm{kV}$ groups was significant $(p<0.01)$. Visual evaluation showed a significant difference between the 100 and $120 \mathrm{kV}$ groups $(p<0.05)$.
\end{abstract}

\section{Keywords}

Low Tube Voltage Technique, Computed Tomography, Acute Ischemic Stroke

\section{Introduction}

Computed tomography (CT) is useful in determining the treatment plan for patients with acute stroke. According to the guidelines of the American Heart Association/American Stroke Association (AHA/ASA), the information necessary to make decisions on emergency measures should be obtained using non-contrast enhanced CT in most cases [1]. CT is useful for excluding cerebral hemorrhage 
and a diagnosis of acute ischemic stroke by identifying early ischemic changes (EIC).

The CT findings indicative of EIC include obscuration of the lentiform nucleus, loss of the insular ribbon, loss of gray-white matter differentiation, and effacement of the cortical sulci. To facilitate identification of these findings, the image should preferably have a high contrast between white matter and gray matter and low noise.

As a tool for increasing the agreement rate between readers, it has been recommended that the Alberta Stroke Program Early CT Score (ASPECT) be used to evaluate for EICs in the middle cerebral artery region [2] [3]. The lower the ASPECT score, the higher the risk of cerebral hemorrhage after treatment with thrombolytic agents [4] [5].

An evaluation method that combines diffusion-weighted imaging (DWI) using magnetic resonance imaging (MRI) and ASPECTS has been reported [6]. MRI alone may result in underestimation of EIC. If the EIC signal on CT is not elevated on DWI, this has been termed reversed discrepancy (RD) [7]. One study identified $\mathrm{RD}$ in $24 \%$ of 164 cases of hyperacute cerebral infarctions within 3 hours of onset and reported that many cases had atrial fibrillation [8].

Evaluation of the vascular occlusion site, collaterals, and penumbra using CT angiography (CTA) and CT perfusion (CTP) is now possible. The usefulness of these modalities in endovascular therapy has been widely reported [9]-[21]. However, while previous studies have reported the utility of low tube voltage imaging for acute ischemic stroke in contrast-enhanced CT [22] [23] [24], to our knowledge no study has evaluated the use of unenhanced CT using low tube voltage.

Therefore, we investigated the accuracy of unenhanced CT using the low tube voltage technique for acute ischemic stroke.

\section{Materials and Methods}

\subsection{Patients and Imaging Protocol}

This retrospective study was approved by the institutional ethics committee of Hyogo College of Medicine (approval number: 2501) and conducted in accordance with the ethical standards of the Declaration of Helsinki. All images used in the study had been acquired as part of routine clinical care during past medical treatment. The purpose of the study and information regarding its implementation were published on the home page of the web site of our institution, and patients were provided the opportunity to opt out.

All patients were scanned using a 128-slice MDCT scanner (Somatom Definition AS+: Edge, Siemens Healthcare, Forchheim Germany). Quantitative evaluation of three groups of 50 patients each was performed at either $120 \mathrm{kV} 500$ mAs, $100 \mathrm{kV} 850 \mathrm{mAs}$, or $100 \mathrm{kV} 750 \mathrm{mAs}$ using sinogram-affirmed iterative reconstruction (SAFIRE). The patient characteristics and scan parameters are shown in Table 1. All included patients were confirmed to not have any lesions, 
including hemorrhage or acute infarction, by neuroradiologists, and their images were studied to quantitatively evaluate the diagnostic performance of the clinical image quality itself. These scan parameters were determined in fundamental physics experiments using a Catphan ${ }^{\circledR} 515$ (The Phantom Laboratory, Salem NY, USA) low-contrast detection module based on $120 \mathrm{kV}$ and visual evaluations by neuroradiologists.

To estimate the CT radiation dose, the CT volume dose index (CTDIvol) and dose length product (DLP) were recorded. The effective dose (ED) for the brain was derived from the product of DLP and a previously reported conversion coefficient for the brain $\left(\mathrm{k}=0.0021 \mathrm{mSv} \cdot \mathrm{mGy}^{-1} \cdot \mathrm{cm}^{-1}\right)$ [25].

\subsection{Image Analysis}

To evaluate image quality under the different protocols, $5 \mathrm{~mm}$ thick images from each reconstruction technique were downloaded from the PACS viewer (Synapse: Fuji Film Medical, Tokyo, Japan) to a workstation (Zio Station2: Ziosoft Inc., Tokyo, Japan). CT values and standard deviation (SD) were measured by setting the region of interest (ROI) as shown in Figure 1. The ROI was set at the putamen and posterior limb of the internal capsule on the axial sections through the basal ganglia to measure CT values of gray and white matter. An ROI with a

Table 1. Patient characteristics and scan parameters.

\begin{tabular}{|c|c|c|c|}
\hline & $120 \mathrm{kV}$ & $100 \mathrm{kV}$ & $100 \mathrm{kV} \mathrm{i}$ \\
\hline \multicolumn{4}{|c|}{ Characteristics } \\
\hline Number of Patients & 50 & 50 & 50 \\
\hline Age & $54.5 \pm 18.1$ & $52.6 \pm 17.7$ & $59.1 \pm 14.6$ \\
\hline Gender (male) & 25 & 26 & 23 \\
\hline Period & $\begin{array}{c}\text { From January } 2011 \\
\text { To April } 2013\end{array}$ & $\begin{array}{c}\text { From May } 2013 \\
\text { To September } 2016\end{array}$ & $\begin{array}{l}\text { From October } 2016 \\
\text { To November } 2018\end{array}$ \\
\hline \multicolumn{4}{|c|}{ Scan parameters } \\
\hline Equipment model & $\mathrm{AS}+$ & $\mathrm{AS}+$ & Edge \\
\hline Tube voltage $(\mathrm{kV})$ & 120 & 100 & 100 \\
\hline Reference mAs & 500 & 850 & 750 \\
\hline Scan method & Non-helical & Non-helical & Volume \\
\hline Beam collimation (mm) & $12 \times 1.2$ & $12 \times 1.2$ & $128 \times 0.6$ \\
\hline kernel & $\begin{array}{c}\text { H37s } \\
\text { medium smooth }\end{array}$ & $\begin{array}{c}\text { H37s } \\
\text { medium smooth }\end{array}$ & $\begin{array}{c}\mathrm{J} 45 \mathrm{~s} \\
\text { medium smooth }\end{array}$ \\
\hline $\begin{array}{l}\text { Hybrid iterative } \\
\text { reconstruction }\end{array}$ & no & no & $\begin{array}{c}\text { SAFIRE } \\
\text { (strength level 3) }\end{array}$ \\
\hline FOV (mm) & 250 & 250 & 250 \\
\hline Scan time (sec) & 2 & 2 & 2 \\
\hline CTDIvol (mGy) & 83.51 & 86.87 & 64.82 \\
\hline
\end{tabular}

FOV: Field of view; CTDI vol: Volume CT dose index. 
Skull base

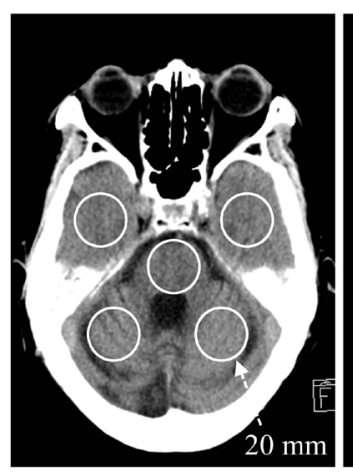

Basal ganglia

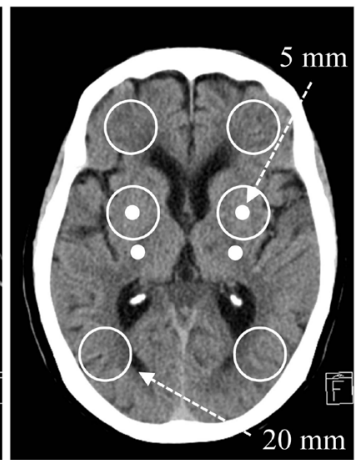

Vertex

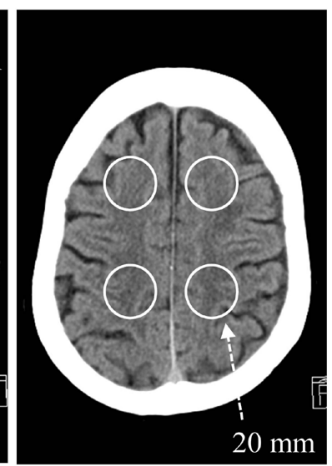

Figure 1. The ROIs were set at the position shown and the SD was measured. ROIs were set at the putamen and posterior limb of the internal capsule to measure the CT value. The diameters of the ROIs are $20 \mathrm{~mm}$ and $5 \mathrm{~mm}$, respectively.

diameter of $20 \mathrm{~mm}$ was used to measure SD, and an ROI with a diameter of 5 $\mathrm{mm}$ was used to measure CT values of gray and white matter.

Image noise was defined as the mean SD of fifteen ROIs. The contrast-to-noise ratio (CNR) was calculated as follows [26]:

$$
\mathrm{CNR}=(\mathrm{CT} \text { valuegray matter }-\mathrm{CT} \text { valuewhite matter }) /(\text { image noise })
$$

\subsection{Statistical Analysis}

Data are expressed as means \pm standard errors of means. Multigroup comparisons were carried out using one-way analysis of variance followed by $\mathrm{Tu}$ key-Kramer multiple comparisons test, and $p<0.01$ was considered statistically significant.

For visual evaluation, five radiological technologists with between 5 and 35 years of experience (mean: 14 years) evaluated the image quality of the brain CT, including image contrast, image noise, and image sharpness. The results obtained were transformed into normalized scores and analyzed using the least significant difference (LSD) method (significance level 5\%) using a normalized rank approach [27]. The five radiological technologists were blinded to the acquisition parameters but were allowed to adjust the window level and width using 2 megapixel color liquid-crystal display monitors (Eizo Nanao Corporation, Ishikawa, Japan).

\section{Result}

A summary of the quantitative evaluation is shown in Table 2. For each imaging protocol, the SD of the skull base, basal ganglia, vertex, and their means are shown in Figure 2. The SD was significantly higher only in the $100 \mathrm{kV}$ group in all cross sections. The mean CT value of the gray and white matter and the increased CT value of the $100 \mathrm{kV}$ group over that of the $120 \mathrm{kV}$ group and contrast are shown in Figure 3 and Figure 4. The rate of increase in CT value was larger for gray matter than for white matter when lowering the tube voltage. A significant 


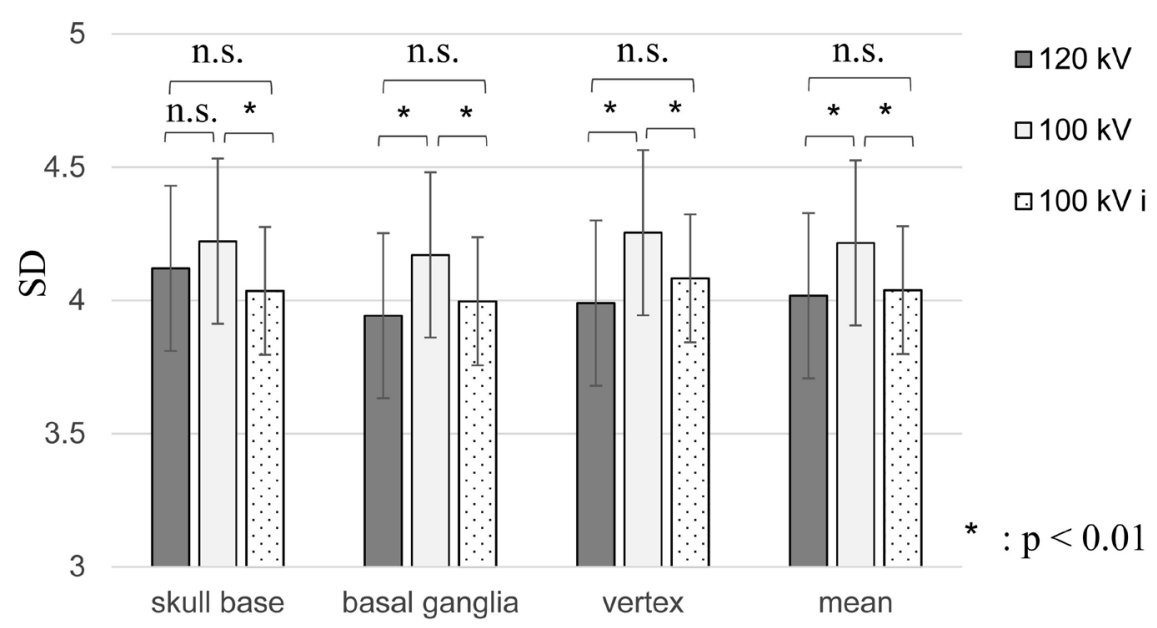

Figure 2. Comparison of the mean SD of the skull base, basal ganglia, and vertex between each imaging protocol. The SD was significantly higher only in the $100 \mathrm{kV}$ group.

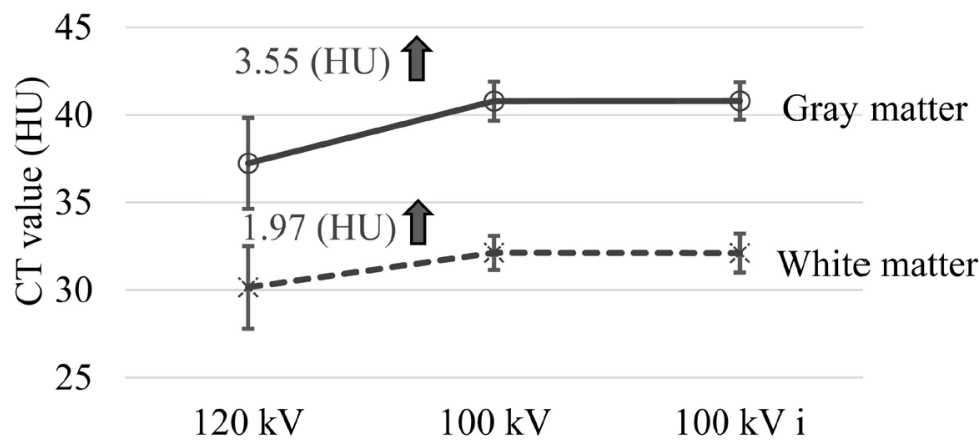

Figure 3. The mean CT value of gray and white matter for each imaging protocol. Note that the CT value of the $100 \mathrm{kV}$ group was significantly higher than that of the $120 \mathrm{kV}$ group. The rate of increase in CT value when lowering the tube voltage was larger for gray matter than for white matter.

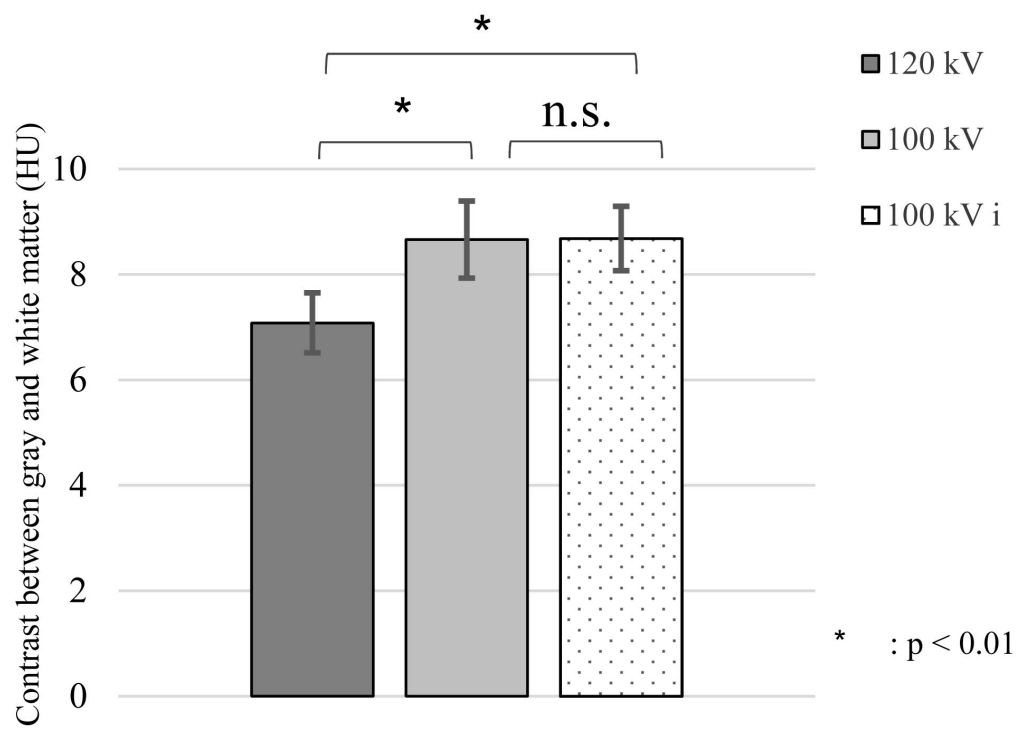

Figure 4. Contrast between gray and white matter. A significant difference was observed between the $100 \mathrm{kV}$ and $120 \mathrm{kV}$ groups. 
difference was observed between the $100 \mathrm{kV}$ and $120 \mathrm{kV}$ groups $(p<0.01)$. The CNR calculated from the contrast between gray and white matter and the mean $\mathrm{SD}$ is shown in Figure 5. A significant difference was observed between the 100 $\mathrm{kV}$ and $120 \mathrm{kV}$ groups $(p<0.01)$. The combined use of SAFIRE and a low tube voltage allowed reduction of the exposure dose. The result of visual evaluation using the normalized ranking method is shown in Figure 6. The LSD was 0.533 . In the case that the distance between the two groups was $>0.533$, there was a significant difference. Therefore, a significant difference was observed between the $100 \mathrm{kV}$ group and $120 \mathrm{kV}$ group $(p<0.05)$.

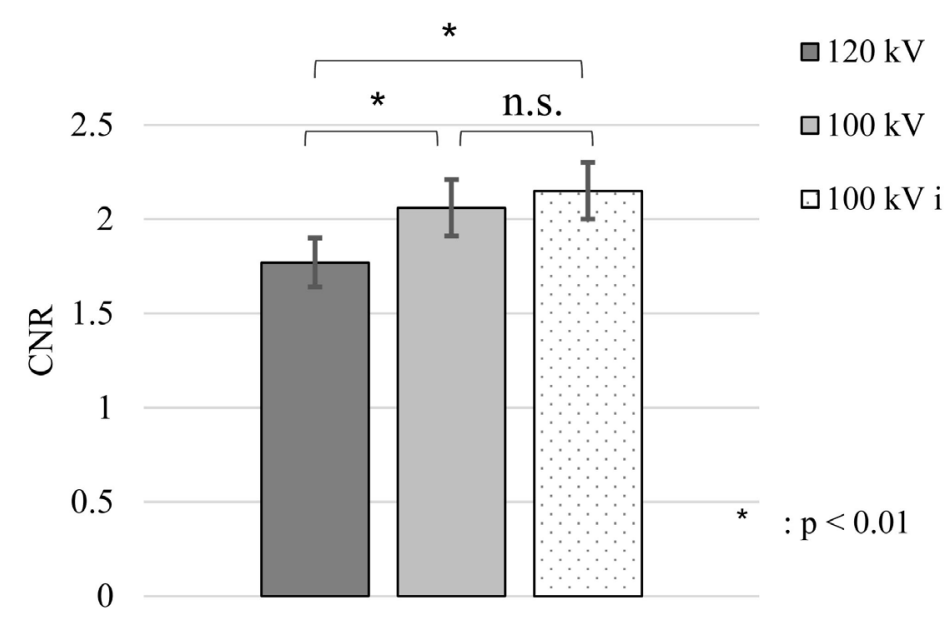

Figure 5. CNR calculated from the contrast between gray and white matter and average of SD. A significant difference was observed between the $100 \mathrm{kV}$ and $120 \mathrm{kV}$ groups.

Table 2. Summary of quantitative evaluation.

\begin{tabular}{|c|c|c|c|}
\hline & $120 \mathrm{kV}$ & $100 \mathrm{kV}$ & $100 \mathrm{kV} \mathrm{i}$ \\
\hline \multicolumn{4}{|c|}{$\mathrm{SD}(\mathrm{HU})$} \\
\hline Skull base & $4.12 \pm 0.36$ & $4.22 \pm 0.31$ & $4.04 \pm 0.33$ \\
\hline Basal ganglia & $3.99 \pm 0.34$ & $4.17 \pm 0.31$ & $4.00 \pm 0.24$ \\
\hline Vertex & $3.99 \pm 0.35$ & $4.25 \pm 0.37$ & $4.08 \pm 0.36$ \\
\hline Average & $4.02 \pm 0.27$ & $4.22 \pm 0.23$ & $4.04 \pm 0.24$ \\
\hline \multicolumn{4}{|c|}{ CT value (HU) } \\
\hline Gray matter & $37.24 \pm 2.59$ & $40.79 \pm 1.07$ & $40.80 \pm 1.12$ \\
\hline White matter & $30.16 \pm 2.36$ & $32.13 \pm 1.11$ & $32.11 \pm 0.97$ \\
\hline Contrast of them & $7.08 \pm 0.56$ & $8.66 \pm 0.73$ & $8.68 \pm 0.61$ \\
\hline $\mathrm{CNR}$ & $1.77 \pm 0.13$ & $2.06 \pm 0.15$ & $2.15 \pm 0.15$ \\
\hline \multicolumn{4}{|c|}{ Output dose (estimated patient exposure dose) } \\
\hline CTDIvol (mGy) & $84.23 \pm 3.87$ & $85.90 \pm 1.46$ & $58.78 \pm 4.33$ \\
\hline $\mathrm{DLP}(\mathrm{mGy} \cdot \mathrm{mm})$ & $1412 \pm 109.1$ & $1481 \pm 143.1$ & $1027 \pm 92.7$ \\
\hline Effective dose (mSv) & $2.97 \pm 0.23$ & $3.09 \pm 0.30$ & $2.16 \pm 0.19$ \\
\hline
\end{tabular}

CNR: Contrast to noise ratio; CTDI vol: Volume CT dose index; DLP: Dose length product. 


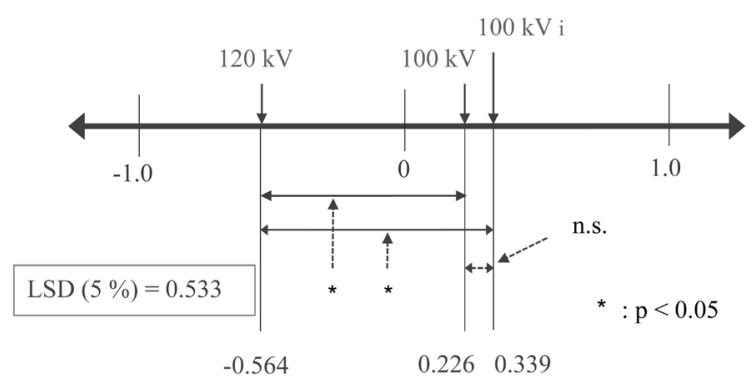

Figure 6. Results of visual evaluation using the normalized ranking method. A significant difference was observed between the $100 \mathrm{kV}$ and $120 \mathrm{kV}$ groups.

\section{Discussions}

Acute ischemic stroke requires urgent assessment of the clinical and radiological features of the brain insult. The ability to exclude hemorrhage and assess the volume of any ischemic tissue to guide therapy allows the proper treatment of acute stroke [28]. A soft-copy review with a variable window width and center level settings was performed in order to improve the detection of parenchymal hypoattenuation of ischemic stroke. Reviewing plain CT images with a narrow window width on a monitor for accentuating the contrast of gray-white matter leads to increased sensitivity for detecting EIC [29]. However, this operation increases image noise. Therefore, if the original image does not improve the contrast between gray and white matter while maintaining image noise, it cannot be said to be useful in cases of acute ischemic stroke.

As a technique to achieve this goal, we focused on low tube voltage. We found that the image noise at $100 \mathrm{kV}$ increased significantly compared to that at 120 $\mathrm{kV}$; however, the contrast between gray and white matter and CNR calculated at these levels was significantly improved, because the rate of increase in CT value when lowering the tube voltage was larger for gray matter than for white matter.

We initially attempted to understand this characteristic using a commercially available phantom, but no phantom separates gray matter and white matter. Development and analysis results of a phantom expressing early ischemic change by changing the density of the substance have been reported [30], but the influence of differences in atomic number on CT value was not considered. Moreover, in Report 44 of the ICRU, the mass attenuation coefficients of gray matter and white matter were equal [31].

Therefore, to discuss why the rate of increase in CT value was larger for gray matter than white matter when lowering the tube voltage, we attempted a simulation using only well-known data without new experiments. The linear attenuation coefficient of the substance at each effective energy was used as clarified in a previous study [32], and the effective energies of $120 \mathrm{kV}$ and $100 \mathrm{kV}$ were identified using the aluminum attenuation method. Figure 7 was obtained by simulating the approximate curve of the CT values of muscle and muscles with varying adipose content at each energy. As the tube voltage decreased, the larger the adipose content, the larger the CT value difference for muscle was shown. Figure 8 adjusted the display range from Figure 7 to the region of interest in this study. 


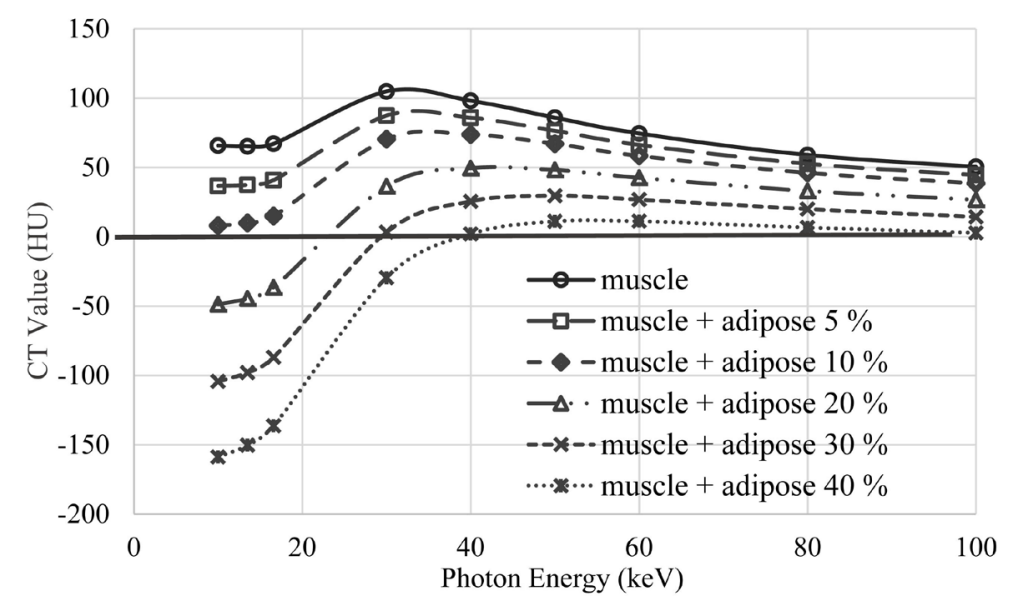

Figure 7. Approximate curve of the CT values of muscle and muscles with varying adipose content at each energy level from a simulation using known linear attenuation coefficient data. As the tube voltage decreased, the larger the adipose content, the larger the CT value difference for the muscle was shown.

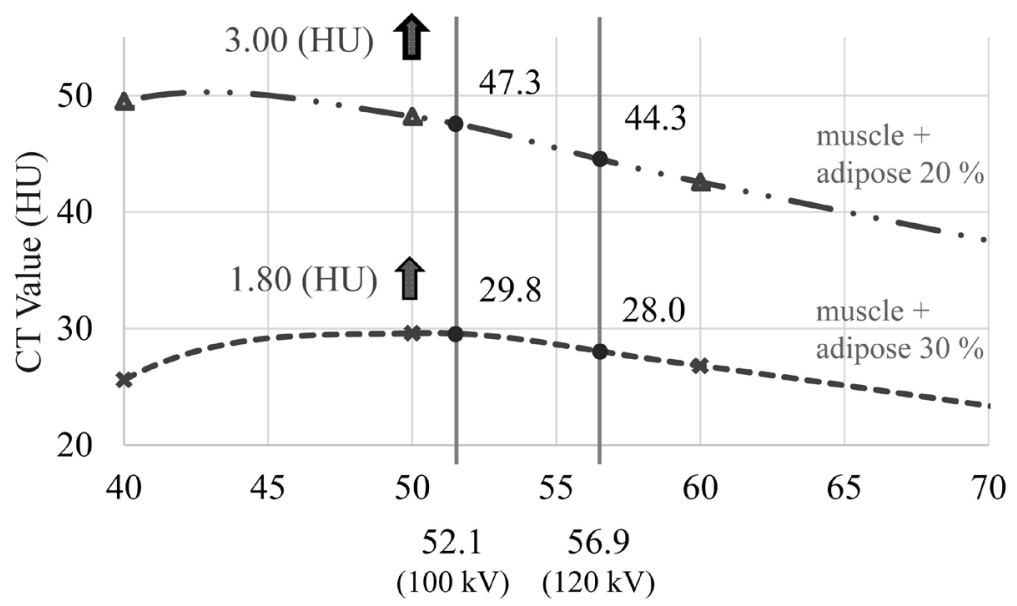

Photon Energy (keV)

Figure 8. This Figure showing Figure 7 limited to the region of interest. The result was obtained by simulating the approximate curve of the CT values of (muscle $+20 \%$ adipose) and (muscle $+30 \%$ adipose) for each energy. CT values at effective energies of 120 $\mathrm{kV}$ and $100 \mathrm{kV}$ are also shown. Assuming gray matter is equivalent to muscle $+20 \%$ adipose and white matter is equivalent to muscle $+30 \%$ adipose, the changes in CT values due to low tube voltage correlated well with results obtained from image analysis.

Assuming gray matter as (muscle $+20 \%$ adipose) and white matter as (muscle + $30 \%$ adipose), the changes in CT values due to low tube voltage correlated well with the results obtained from image analysis.

The ideas obtained from the simulation can be explained by the following two points. First, as the adipose content increases, the effective atomic number decreases. Second, as the tube voltage decreases and the atomic number increases, the cross section of photoelectric absorption exponentially increases [33]. Therefore, we considered that white matter, covered with myelin, contains significant adipose, so that the change in CT value due to the change in tube vol- 
tage was smaller than that for gray matter.

This study has several limitations. All imaging data were obtained with a single CT device. Even at the same tube voltage, differences among equipment manufacturers mean that the energy spectrum differs; therefore, devices with lower effective energy may show results discrepant with our results due to the greater influence of beam hardening.

Moreover, since hybrid iterative reconstruction (HIR) is a nonlinear image processing method, a previous study recommended that image noise should be evaluated using the noise-power spectrum (NPS) instead of SD [34]; however, calculating CNR using NPS did not change the finding of a significant difference in this study. Furthermore, a setting of $100 \mathrm{kV}$ using SAFIRE could reduce image noise while maintaining improved contrast between gray and white matter, and its estimated patient exposure dose was lower than that of other methods. However, the HIR algorithm could not improve low-contrast detectability over that achieved with filtered back projection at the same radiation doses [34]; therefore, we considered that a sufficient dose (i.e. SD of the brain parenchyma is 4 or less at a slice thickness of $5 \mathrm{~mm}$, with reference to GALACTIC by the Japanese Society of Radiological Technology) was necessary to evaluate EIC to the same extent.

\section{Conclusion}

CT with an adequate exposure dose using a low tube voltage technique can effectively utilize the difference in adipose content between gray and white matter to improve image quality required in cases of acute ischemic stroke with or without SAFIRE.

\section{Acknowledgements}

We thank Libby Cone, MD, MA, from DMC Corp. (http://dmed.co.jp/) for editing a draft of this manuscript.

\section{Research Involving Human Participants and/or Animals}

All procedures in studies involving human participants were performed in accordance with the ethical standards of Hyogo College of Medicine, and with the 1964 Helsinki Declaration and its later amendments or comparable ethical standards. This article does not contain any studies with animals performed by any of the authors.

\section{Informed Consent}

The purpose of the research and information on its implementation were published on the home page of the web site of our institution, and patients were provided the opportunity to opt out.

\section{Presentation at Conferences}

This work was presented in part at the 33rd Japan Conference of Radiological 
Technologists, in Hakodate, Japan, on 22-24 September, 2017.

\section{Conflicts of Interest}

The authors declare no conflicts of interest regarding the publication of this paper.

\section{References}

[1] Powers, W.J., Derdeyn, C.P., Biller, J., et al. (2015) AHA/ASA Focused Update of the 2013 Guidelines for the Early Management of Patients with Acute Ischemic Stroke Regarding Endovascular Treatment: A Guideline for Healthcare Professionals from the American Heart Association/American Stroke Association. Stroke, 46, 3020-3035. https://doi.org/10.1161/STROKEAHA.115.010716

[2] Barber, P.A., Demchuk, A.M., Zhang, J., et al. (2000) Validity and Reliability of a Quantitative Computed Tomography Score in Predicting Outcome of Hyperacute Stroke before Thrombolytic Therapy. ASPECTS Study Group. The Lancet, 355, 1670-1674. https://doi.org/10.1016/S0140-6736(00)02237-6

[3] Pexman, J.H., Barber, P.A., Hill, M.D., et al. (2001) Use of the Alberta Stroke Program Early CT Score (ASPECTS) for Assessing CT Scans in Patients with Acute Stroke. American Journal of Neuroradiology, 22, 1534-1542.

[4] Dzailowski, I., Hill, M.D., Coutts, S.B., et al. (2006) Extend of Early Ischemic Changes on Computed Tomography (CT) before Thrombolysis: Prognostic Value of the Alberta Stroke Program Early CT Score in ECASS II. Stroke, 37, 973-978. https://doi.org/10.1161/01.STR.0000206215.62441.56

[5] Hirano, T., Sasaki, M., Tomura, N., et al. (2012) Japan Alteplase Clinical Trial Group: Low Alberta Stroke Program Early Computed Tomography Score within 3 Hours of Onset Predicts Subsequent Symptomatic Intracranial Hemorrhage in $\mathrm{Pa}$ tients Treated with $0.6 \mathrm{mg} / \mathrm{kg}$ Alteplase. Journal of Stroke and Cerebrovascular Diseases, 21, 898-902. https://doi.org/10.1016/j.jstrokecerebrovasdis.2011.05.018

[6] Nezu, T., Koga, M., Nakagawara, J., et al. (2011) Early Ischemic Change on CT Versus Diffusion-Weighted Imaging for Patients with Stroke Receiving Intravenous Recombinant Tissue-Type Plasminogen Activator Therapy: Stroke Acute Management with Urgent Risk-Factor Assessment and Improvement (SAMURAI) rt-PA Registry. Stroke, 42, 2196-2200. https://doi.org/10.1161/STROKEAHA.111.614404

[7] Kim, E.Y., Ryoo, J.W., Roh, H.G., et al. (2006) Reversed Discrepancy between CT and Diffusion-Weighted MR Imaging in Acute Ischemic Stroke. American Journal of Neuroradiology, 27, 1990-1995.

[8] Kawano, H., Hirano, T., Nakajima, M., et al. (2013) Diffusion-Weighted Magnetic Resonance Imaging May Underestimate Acute Ischemic Lesions: Cautions on Neglecting a Computed Tomography-Diffusion-Weighted Imaging Discrepancy. Stroke, 44, 1056-1061. https://doi.org/10.1161/STROKEAHA.111.000254

[9] Puetz, V., Dzialowski, I., Hill, M.D., et al. (2008) Calgary CTA Study Group: Intracranial Thrombus Extent Predicts Clinical Outcome, Final Infarct Size and Hemorrhagic Transformation in Ischemic Stroke: The Clot Burden Score. International Journal of Stroke, 3, 230-236. https://doi.org/10.1111/j.1747-4949.2008.00221.x

[10] Sillanpää, N., Saarinen, J.T., Rusanen, H., et al. (2012) Location of the Clot and Outcome of Perfusion Defects in Acute Anterior Circulation Stroke Treated with Intravenous Thrombolysis. American Journal of Neuroradiology, 34, 100-106. https://doi.org/10.3174/ajnr.A3149 
[11] Tan, I.Y., Demchuk, A.M., Hopyan, J., et al. (2009) CT Angiography Clot Burden Score and Collateral Score: Correlation with Clinical and Radiologic Outcomes in Acute Middle Cerebral Artery Infarct. American Journal of Neuroradiology, 30, 525-531. https://doi.org/10.3174/ajnr.A1408

[12] Thierfelder, K.M., Sommer, W.H., Ertl-Wagner, B., et al. (2016) Prediction of Stent-Retriever Thrombectomy Outcomes by Dynamic Multidetector CT Angiography in Patients with Acute Carotid T or MCA Occlusions. American Journal of Neuroradiology, 37, 1296-1302. https://doi.org/10.3174/ajnr.A4694

[13] Menon, B.K., Qazi, E., Nambiar, V., et al. (2015) IMS Investigators: Differential Effect of Baseline CTA Collaterals on Clinical Outcome in Patients Enrolled in the IMS-III Trial. Stroke, 46, 1239-1244. https://doi.org/10.1161/STROKEAHA.115.009009

[14] Nambiar, V., Sohn, S.I., Almekhlafi, M.A., et al. (2014) CTA Collateral Status and Response to Recanalization in Patients with Acute Ischemic Stroke. American Journal of Neuroradiology, 35, 884-890. https://doi.org/10.3174/ajnr.A3817

[15] Souza, L.C., Yoo, A.J., Chaudhry, Z.A., et al. (2012) Malignant CTA Collateral Profile Is Highly Specific for Large Admission DWI Infarct Core and Poor Outcome in Acute Stroke. American Journal of Neuroradiology, 33, 1331-1336. https://doi.org/10.3174/ajnr.A2985

[16] Menon, B.K., d'Esterre, C.D., Qazi, E.M., et al. (2015) Multiphase CT Angiography: A New Tool for the Imaging Triage of Patients with Acute Ischemic Stroke. Radiology, 275, 510-520. https://doi.org/10.1148/radiol.15142256

[17] Yan den Wijngaard, I.R., Holswilder, G., Wermer, M.J., et al. (2016) Assessment of Collateral Status by Dynamic CT Angiography in Acute MCA Stroke: Timing of Acquisition and Relationship with Final Infarct Volume. American Journal of Neuroradiology, 37, 1231-1236. https://doi.org/10.3174/ajnr.A4746

[18] Lin, L., Bivard, A., Krishnamurthy, V., et al. (2016) Whole-Brain CT Perfusion to Quantify Acute Ischemic Penumbra and Core. Radiology, 279, 876-887. https://doi.org/10.1148/radiol.2015150319

[19] Lansberg, M.G., Christensen, S., Kemp, S., et al. (2017) CT Perfusion to Predict Response to Recanalization in Ischemic Stroke Project (CRISP) Investigators: Computed Tomographic Perfusion to Predict Response to Recanalization in Ischemic Stroke. Annals of Neurology, 81, 849-856. https://doi.org/10.1002/ana.24953

[20] Campbell, B.C., Christensen, S., Levi, C.R., et al. (2011) Cerebral Blood Flow Is the Optimal CT Perfusion Parameter for Assessing Infarct Core. Stroke, 42, 3435-3440. https://doi.org/10.1161/STROKEAHA.111.618355

[21] Inoue, M., Mlynash, M., Straka, M., et al. (2012) Patients with the Malignant Profile within 3 Hours of Symptom Onset Have Very Poor Outcomes after Intravenous Tissue-Type Plasminogen Activator Therapy. Stroke, 43, 2494-2496.

https://doi.org/10.1161/STROKEAHA.112.653329

[22] Waaijer, A., Prokop, M., Velthuis, B.K., Bakker, C.J.G., et al. (2007) Circle of Willis at CT Angiography: Dose Reduction and Image Quality-Reducing Tube Voltage and Increasing Tube Current Settings. Radiology, 242, 832-839. https://doi.org/10.1148/radiol.2423051191

[23] Luo, S., Zhang, L.J., Meinel, F.G., et al. (2014) Low Tube Voltage and Low Contrast Material Volume Cerebral CT Angiography. European Radiology, 24, 1677-1685. https://doi.org/10.1007/s00330-014-3184-z

[24] Cho, C.S., Chung, T.S., Oh, D.K., et al. (2012) Cerebral Computed Tomography Angiography Using a Low Tube Voltage $(80 \mathrm{kVp})$ and a Moderate Concentration of Iodine 
Contrast Material: A Quantitative and Qualitative Comparison with Conventional Computed Tomography Angiography. Investigative Radiology, 47, 142-147. https://doi.org/10.1097/RLI.0b013e31823076a4

[25] Huda, W., Ogden, K.M., Khorasani, M.R., et al. (2008) Converting Dose Length Product to Effective Dose at CT. Radiology, 248, 995-1003. https://doi.org/10.1148/radiol.2483071964

[26] Gupta, A.K., Nelson, R.C., Johnson, G.A., et al. (2003) Optimization of Eight-Element Multi-Detecter Row Helical CT Technology for Evaluation of the Abdomen. Radiology, 227, 739-745. https://doi.org/10.1148/radiol.2273020591

[27] Nakamae, M. (2000) Study of the Reliability of Visual Evaluation by the Ranking Method Analysis of Ordinal Scale and Psychological Scaling Using the Normalized Rank Approach. Japanese Journal of Radiological Technology, 56, 725-730. https://doi.org/10.6009/jjrt.KJ00001356925

[28] von Kummer, R., Bourquain, H., Bastianello, S., Bozzao, L., Manelfe, C., Meier, D. and Hacke, W. (2001) Early Prediction of Irreversible Brain Damage after Ischemic Stroke at CT. Radiology, 219, 95-100.

https://doi.org/10.1148/radiology.219.1.r01ap0695

[29] Lev, M.H., Farkas, J., Gemmete, J.J., Hossain, S.T., Hunter, G.J., Koroshetz, W.J., et al. (1999) Acute Stroke: Improved Nonenhanced CT Detection-Benefits of Soft-Copy Interpretation by Using Variable Window Width and Center Level Settings. Radiology, 213, 150-155. https://doi.org/10.1148/radiology.213.1.r99oc10150

[30] Nishimura, K., Tanaka, C., Fukao, M., et al. (2016) The Detectability of Iterative CT Reconstruction for Low-Contrast Lesions in Hyperacute Cerebral Infarction: Assessment with Newly Developed Dedicated Head Phantoms. Japanese Journal of Radiological Technology, 72, 157-164. https://doi.org/10.6009/jjrt.2016_JSRT_72.2.157

[31] Goldstone, K.E. (1990) Tissue Substitutes in Radiation Dosimetry and Measurement. ICRU Report 44, International Commission on Radiation Units and Measurements, 41 .

[32] Shirotani, T. (1995) Attenuation Coefficients of Human Tissues and Tissue Substitutes. Japan Atomic Energy Research Institute, Ibaraki, 95-002.

[33] Hubbell, J.H., et al. (1969) Photon Cross Sections, Attenuation Coefficients and Energy Absorption Coefficients from $10 \mathrm{keV}$ to $100 \mathrm{GeV}$. Natl. Stand. Ref. Data, 29. https://doi.org/10.6028/NBS.NSRDS.29

[34] Urikura, A., Hara, T., Ichikawa, K., et al. (2016) Objective Assessment of Low-Contrast Computed Tomography Images with Iterative Reconstruction. Physica Medica, 32, 992-998. https://doi.org/10.1016/j.ejmp.2016.07.003 